\title{
Improving DPA by Peak Distribution Analysis
}

\author{
Jing $\operatorname{Pan}^{1}$, Jasper G.J. van Woudenberg ${ }^{1}$, Jerry I. den Hartog ${ }^{2}$, \\ and Marc F. Witteman ${ }^{1}$ \\ 1 Riscure BV, 2628 XJ Delft, The Netherlands \\ $\{$ pan, vanwoudenberg, witteman\} @riscure.com \\ 2 Eindhoven University of Technology, \\ 5600 MB Eindhoven, The Netherlands \\ j.d.hartog@tue.nl
}

\begin{abstract}
Differential Power Analysis (DPA) attacks extract secret key information from cryptographic devices by comparing power consumption with predicted values based on key candidates and looking for peaks which indicate a correct prediction. A general obstacle in the use of DPA is the occurrence of so called ghost peaks, which may appear when evaluating incorrect key candidates. Some ghost peaks can be expected from the structure and may actually leak information. We introduce a DPA enhancement technique-Euclidean Differential Power Analysis (EDPA), which makes use of the information leaked by the ghost peaks to diminish the ghost peaks themselves and bring forward the correct key candidate. The EDPA can be combined with any standard DPA attack irrespective of the distinguisher used. We illustrate that EDPA improves on DPA with both simulations and experiments on smart cards.
\end{abstract}

Keywords: Differential power analysis, Euclidean similarity, ghost peaks.

\section{Introduction}

Side-channel attacks (SCA) reveal the secret key of a cryptosystem based on information gained from physical implementation of the cryptosystem on a smart card or other device. Information provided by sources such as timing [8], power consumption [9] and electromagnetic emulation [15] can be exploited by SCA attacks to break cryptosystems. Differential power analysis (DPA) 9] is a form of SCA that can extract secrets from power consumption measurements which may contain a lot of noise. Using DPA, an adversary can obtain intermediate values within cryptographic computations by statistically analyzing the power consumption measurements collected from multiple cryptographic operations performed by a vulnerable device.

A successful DPA attack would typically result in a vector of statistical test results amongst which the highest value occurs for the correct key candidate and the correct time (the moment in time during which the examined intermediate value is manipulated on the device). This highest value is usually referred to as the correct peak and the location in the vector where this peak occurs as the correct time. An unsuccessful attack normally implies a 'ghost peak' problem,

A. Biryukov, G. Gong, and D.R. Stinson (Eds.): SAC 2010, LNCS 6544, pp. 241 261, 2011.

(C) Springer-Verlag Berlin Heidelberg 2011 
where the highest peak does not accord to the correct key candidate and thus erroneous conclusions can be drawn from the attack results.

Both academic research and practical experimentation have been conducted to make a clearer distinction between the correct peak and the ghost peaks resulted from a DPA attack. Proposed solutions include, for example, improvement on measurement techniques such that strong noises are filtered out from the power traces [13, improvement on the trace alignment such that power signals that correspond to the same intermediate result are located at the same position for all the traces [18, and improvement on DPA algorithms so that suitable power models or statistic tools are applied in specific attacks [2 5]. Such solutions attempt to reduce noise but at best can achieve the theoretical distinctions between the correct peak and the ghost peaks obtained in an attack using noisefree measurements. No solutions have, to the best of our knowledge, involved strengthening the correct peak by analyzing the distribution of the DPA results of all key hypotheses.

Here we propose a novel approach which treats the ghost peaks as a source of information as well and allows an attack to reduce ghost peaks based on information provided by the ghost peaks themselves. The proposed approach is built upon a standard DPA attack with additional adjustment made as suggested by the characteristics of the ghost peaks. Typically, the distribution of the DPA results at the correct time shows a predictable pattern with the real key giving the highest peak and some of the incorrect keys giving ghost peaks. Our attack exploits this information by comparing the distribution of the peaks in a real attack to the distribution of the peaks predicted by a hypothetical noise free attack. We use Euclidean similarity [6] to match the hypothesis with the actually observed pattern of peaks and hence refer to this method as the Euclidean Differential Power Analysis (EDPA).

The distribution of the DPA results at the correct time will always reflect the correct key. The DPA attack, however, only looks which peak is the highest and ignores the distribution of other peaks. Hence, there is always additional information about the key that an EDPA attack regards and a DPA attack ignores. We apply EDPA to Correlation Power Analysis (CPA) [2, calling the resulting method ECPA, and show that ECPA improves on CPA, almost always providing a clearer distinction between the correct peak and ghost peaks. We show this using both simulation and physical experiments on smart cards.

Commonly used measures to evaluate the effectiveness of SCA attacks, so called SCA security metrics, are success rate and guessing entropy [17. As an additional result we propose a new measure 'oth-order guessing entropy' and argue that it is more practical than the original guessing entropy.

The remainder of this paper is organized as follows. Section 2 recaps success rate and guessing entropy, analyzes their relation and introduces oth-order guessing entropy. Section 3 explains an EDPA attack in more detail. Section 4 demonstrates ECPA in simulation and compares it to CPA. In Section 5, the two attacks are applied and compared using experiments on smart cards. Finally, Section 6 provides conclusions. 


\section{SCA Security Metrics}

Soon after the introduction of SCA attacks [8] many different attacks and optimizations of attacks were proposed e.g. 12112. To be able to compare different attacks several SCA security metrics have been proposed such as success rate [17, guessing entropy [17, signal-to-noise ratios [12] and number of traces needed [3]. How meaningful a metric is and how easy it is to evaluate depends a lot on the type of adversary and the conditions under which the attack is performed. Thus often several metrics are combined when evaluating a SCA attack, see e.g. [1016].

Here we have chosen to use $o$-th order success rate (SR in short) and guessing entropy (GE in short) as our security metrics. SR makes sense if the adversary only looks at the top results. GE is compatible if the adversary only uses the DPA as a method to sort key candidates for a brute-force attack. Additionally, we introduce a new security metric, called oth-order guessing entropy, which is similar to guessing entropy but better reflects the fact that the computational ability of an attacker will have some limit. Below we first quickly recall success rate and guessing entropy and then discuss the need for and define $o$-th order guessing entropy.

\subsection{Success Rate and Guessing Entropy}

An SCA attack executes a set of queries, obtains side channel information during the execution 11 and based on this information sorts key candidates according to their likelihood of being correct. Let random variable $\mathbf{g}_{q}$ denote the vector resulting from sorting all possible key candidates based on an attack using $q$ queries. (Note that in practice $\mathbf{g}_{q}$ is built by combining the results from many sub-SCA, where each sub-attack targets a different sub-key. See also Section 2.2) The success rate of order $o, s r_{\mathbf{g}_{q}}^{o}$, is the probability that the correct key $s$ is ranked amongst the first $o$ candidates in attack $\mathbf{g}_{q}$. The guessing entropy, $g e_{\mathbf{g}_{q}}$, gives the expected index of the correct key in $\mathbf{g}_{q}$. Intuitively, $\mathrm{SR}$ measures the probability of success with a fixed limit on the amount of computation, while GE measures the average cost of a brute-force key search with no limit on the computational ability.

Note that the GE of an attack can be calculated based on the SR of the attack for the various orders of SR. For $S=\left|\mathbf{g}_{q}\right|$ and $i=1,2, \ldots, S$ let $\mathcal{P}\left(\mathbf{g}_{q}[i]=s\right)$ denote the probability that the correct key $s$ is ranked at position $i$ in $\mathbf{g}_{q}$, then

$$
\begin{aligned}
& s r_{\mathbf{g}_{q}}^{0}=0, \\
& s r_{\mathbf{g}_{q}}^{i}=s r_{\mathbf{g}_{q}}^{i-1}+\mathcal{P}\left(\mathbf{g}_{q}[i]=s\right), \\
& g e_{\mathbf{g}_{q}}=\sum_{i=1}^{S} i \cdot \mathcal{P}\left(\mathbf{g}_{q}[i]=s\right)=\sum_{i=1}^{S} i \cdot\left(s r_{\mathbf{g}_{q}}^{i}-s r_{\mathbf{g}_{q}}^{i-1}\right) .
\end{aligned}
$$

\footnotetext{
${ }^{1}$ This may involve optimizations to reduce noise such as running the algorithm multiple times with the same input and averaging the measurements.
} 


\subsection{Sorting Key Candidates}

An SCA attack divides a secret key into several sub-keys each of which contains a small number of bits. A series of sub-SCA attacks are applied to rate the likelihood of each possible sub-key. The full keys are then sorted and tested in an order derived from the likelihood of the sub-keys. It is reasonable to assume that, to improve the attack, an adversary will not just use the ranking in the sub-attacks for sorting full keys but will also perform some global optimization combining and comparing ratings across different sub-attacks.

For independent sub-attacks combined with naive sorting, the product of GEs can be used as an approximation of the full GE. When using global optimizations there is no guarantee this is still a good estimation. Only when there is a consistent and similar improvement in GE across the sub-attacks is it reasonable to assume this translates into a proportional advantage in the overall GE. (See also Section 4.3.)

Without loss of generality, we focus on two sorting algorithms that can be used to sort the key candidates in $\mathbf{g}_{q}$. The first is an optimal solution, which performs a full global-optimization by sorting the key candidates in descending order of their combined sub-SCA results. The cost of sorting $(O(N \log (N))$ where $N$ is the size of the key space) is acceptable compared to the effort needed for a brute-force attack, so an attacker will likely want to do this to optimize attack. However, note that $N$ is exponential in the key size $\left(N=2^{|k|}\right)$ which means that actually doing the sorting will quickly become unfeasible. This means that estimating the guessing entropy with experiments is not realistically possible.

We address this problem in two ways. The first is to introduce a second, suboptimal, sorting algorithm. It sorts the key candidates by a heuristic search in which optimal next key candidates are only selected at fixed points in the sorting rather than at every step. With this algorithm we are able to calculate the index of the correct key (in time polynomial in the key size) without actually having to do the sorting. However, the index of a key may increase a lot compared to its optimal ranking which may, especially for very noisy sub-SCA results, cause a much higher index of the correct key. (We refer the interested readers to Appendix $\mathrm{A}$ for a more detailed description of the sorting algorithms.)

The second way we address the problem with computing guessing entropy is to propose an enhanced alternative security measure called $o$-th order guessing entropy.

\subsection{The oth-Order Guessing Entropy Security Metric}

The GE of an attack measures the average brute-force cost after an SCA attack without a limit on the amount of computation. In the other words, it assumes that the adversary can always test as many key candidates as it is necessary to find the correct key. This is usually not a practical assumption. Considering the usually large key space (56-bit DES key or 128-bit AES key) an adversary will likely have to limit the number of candidates tested before considering the attack a failure. To capture this in a security measure, we introduce $o$-th order GE. 


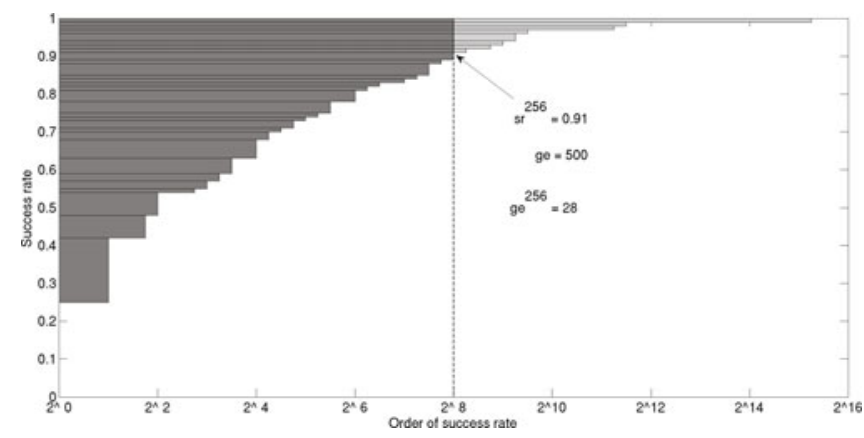

Fig. 1. An example of $s r_{\mathbf{g}_{q}}^{o}, g e_{\mathbf{g}_{q}}$ and $g e_{\mathbf{g}_{q}}^{o}$

The oth-order guessing entropy of an attack $\mathbf{g}_{q}$, written as $g e_{\mathbf{g}_{q}}^{o}$, measures the average number of key candidates to test after an SCA attack with $q$ queries, given that in maximum $o$ candidates can be tested. Intuitively, it merges the concept of the 'average cost' from the guessing entropy and the 'maximum cost limit' from the success rate. It allows an easily interpretable measurement when characterizing the average cost of a post-SCA brute-force attack in practical scenarios where the adversary does not test all possible key candidates.

Like $g e_{\mathbf{g}_{q}}$, the $g e_{\mathbf{g}_{q}}^{o}$ of an attack can be calculated from the success rates $s r_{\mathbf{g}_{q}}^{o}$ of the attack for the various order $o$ :

$$
\begin{aligned}
g e_{\mathbf{g}_{q}}^{o} & =\sum_{i=1}^{o} i \cdot \mathcal{P}\left(\mathbf{g}_{q}[i]=s\right)+o \cdot \sum_{i=o+1}^{S} \mathcal{P}\left(\mathbf{g}_{q}[i]=s\right) \\
& =\sum_{i=1}^{o} i \cdot\left(s r_{\mathbf{g}_{q}}^{i}-s r_{\mathbf{g}_{q}}^{i-1}\right)+o \cdot\left(1-s r_{\mathbf{g}_{q}}^{o}\right) .
\end{aligned}
$$

Figure1illustrates the relation between $s r_{\mathbf{g}_{q}}^{o}, g e_{\mathbf{g}_{q}}$ and $g e_{\mathbf{g}_{q}}^{o}$ using results from a simulated CPA attack that targets eight DES S-boxes (see Section 4.2). The curve formed by the bottom of the colored area depicts the $s r^{o}$ for various $o$. The (dark and light) gray region visualizes the calculation of the ge based on Eq. (1) with each of the horizontal bars marking an area equal to $i \cdot\left(s r^{i}-s r^{i-1}\right)$ for some order $i$. As the summation of those small bars, the $g e$ is then equal to the entire gray area. The oth-order guessing entropy, $g e^{o}$, limits the maximum computation to $o$ and thus is equal to the dark gray area between the axis $x=0$ and the line $x=o$. Compared to ge, the light gray area to the right of the line $x=o$ is omitted from $g e^{o}$. (Note that this area is much larger than it appears due to the logarithmic scale.)

\section{The EDPA Attack}

The result of a DPA attack $\mathcal{S}$ is calculated based on some distinguisher $\mathcal{D}$ that compares the real power measurement $\mathcal{T}$ to the hypothetical power 
consumption values $\mathcal{H}: \mathcal{S}=\mathcal{D}(\mathcal{T}, \mathcal{H})$. The distinguisher $\mathcal{D}$ can be various statistical tests, such as difference-of-means [9], Pearson's correlation coefficient [2], mutual information [7, variance test 16, etc. An EDPA attack can be built on top of any DPA attack independently from the distinguisher used. In this section, we demonstrate the construction of an EDPA attack with emphasis on ECPA, where Pearson's correlation coefficient is used as the distinguisher $\mathcal{D}$. Note that all attacks mentioned in this section are actually sub-attacks using the terminology of Section 2 .

\subsection{Description of the Attack}

We use the block representations of [11, Ch. 6] to describe our attack. (See appendix Bfor a graphical representation.) The hypothetical power consumption values are contained in a $q \times n$ matrix $\mathbf{H}$, with $q$ being the number of queries used and $n$ being the number of key candidates in this attack. A column of $\mathbf{H}$, written as $\boldsymbol{h}_{i}$, corresponds to a key hypothesis $k_{i}$. The result of a DPA attack can be represented as an $n \times m$ matrix $\mathbf{S}$, calculated based on $n$ key hypotheses and $m$ time samples. In $\mathbf{S}, s_{i, j}$ corresponds to the DPA result for key hypothesis $k_{i}$ and time sample $t_{j}$. Let $(c k, c t)$ be the index of the correct peak in $\mathbf{S}$, i.e. $k_{c k}$ is the correct key and $t_{c t}$ is the correct time.

After obtaining the results of DPA, an EDPA attack continues with the following three steps.

Step 1: Generate hypothetical attack results. The hypothetical power consumption values of a DPA attack model the power consumption of the device caused by the processing of the targeted intermediate value. We use this model to simulate the attack with each of the $n$ key candidate as the correct key. As each attack gives a result for every key candidate this yields an $n$-by- $n$ matrix $\mathbf{C}$ of DPA results, where $c_{i, j}=\mathcal{D}\left(\boldsymbol{h}_{j}, \boldsymbol{h}_{i}\right)$. The $c k$-th column $\boldsymbol{c}_{c k}$ in $\mathbf{C}$ thus models the expected DPA results at the correct time ct. For ECPA this means that, for candidate $k_{i}$ given correct key $k_{j}$, we calculate the correlation between columns $\boldsymbol{h}_{j}$ and $\boldsymbol{h}_{i}$ of $\mathbf{H}, c_{i, j}=\rho\left(\boldsymbol{h}_{j}, \boldsymbol{h}_{i}\right)(i, j=1 . . n)$. In this case we refer to $\mathbf{C}$ as inter-data correlation.

Step 2: Compare the actual and the hypothetical attacks. Next we match the DPA results predicted for each key candidate (i.e. each column of $\mathbf{C}$ ) with the actually observed DPA results at each point in time (i.e. each column of $\mathbf{S}$ ) using Euclidean similarity [6. In ECPA a similarity $e_{i, j}$ is assigned to column $i$ of the inter-data correlation $\mathbf{C}$ and column $j$ of DPA results $\mathbf{S}$, as defined in Eq. (11) $(i=1 . . n$ and $j=1 . . m)$ resulting in an $n \times m$ matrix E. Like in $\mathbf{S}, e_{i, j}$ corresponds to key hypothesis $k_{i}$ and time sample $t_{j}$.

$$
e_{i, j}=1-\frac{d_{i, j}}{\max \left\{d_{x, j} \mid x=1 . . n\right\}}, \quad \text { where } \quad d_{i, j}=\sqrt{\sum_{p=1}^{n}\left(c_{p, i}-s_{p, j}\right)^{2}} .
$$

Here $d_{i, j}$ is the Euclidean distance between vectors $\boldsymbol{c}_{i}$ and $\boldsymbol{s}_{j}$. 


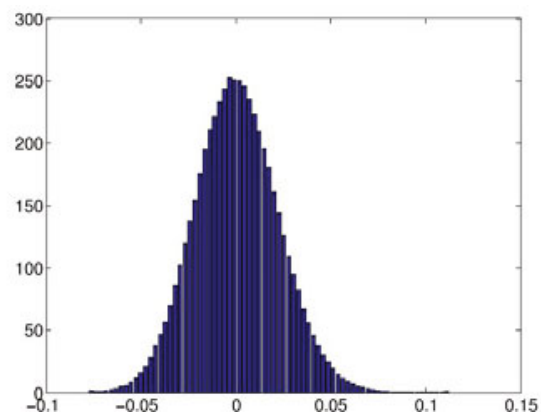

Fig. 2. Histogram of the distribution of $\mathbf{S}$

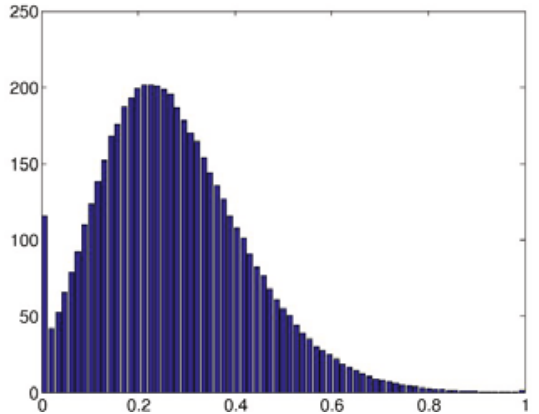

Fig. 3. Histogram of the distribution of $\hat{\mathbf{E}}$

Step 3: Combine the DPA and Euclidean similarity results. Finally, the DPA $\mathbf{S}$ is scaled using the Euclidean similarity $\mathbf{E}$ by Eq. (2) resulting the final result $\mathbf{R}$ of the attack.

$$
r_{i, j}=\frac{1}{2} s_{i, j}+\frac{1}{2} s_{i, j} \cdot \hat{e}_{i, j}, \quad \text { where } \quad \hat{e}_{i, j}=\frac{e_{i, j}}{\max \left\{e_{x, y} \mid x=1 . . n, y=1 . . m\right\}} .
$$

The highest value in $\mathbf{R}$ now reveals the location $(c k, c t)$ and hence $k_{c k}$ and $t_{c t}$. Let $\hat{\mathbf{E}}$ be the matrix of the normalized Euclidean similarity $\hat{e}_{i, j}$. The mixing function Eq. (2) scales down the DPA values by factors of 0 to $1 / 2$, linearly to the corresponding normalized Euclidean similarity: $s_{i, j}$ remains unaltered if $\hat{e}_{i, j}=1$ and $s_{i, j}$ is halved if $\hat{e}_{i, j}=0$.

Remark 1. In contrast to DPA attacks where only the highest correlation value is used to indicate the correct key hypothesis, EDPA attacks determine the correct key based on the correlation values of all key candidates. As presence of DPA peaks where they are expected more strongly indicates the correct key than absence of DPA peaks where none is expected, Euclidean similarity is used to ensure that higher DPA values contribute more than lower ones. The Euclidean similarity values are then used to tune the DPA values such that only the peaks that are significant in both are retained.

Remark 2. In choosing the mixing function in Eq. (2) we note that the Euclidean similarity measure is more sensitive to noise, as illustrated in Figures 2 and 3 . These figures plot distributions of the DPA results $\mathbf{S}$ and the normalized Euclidean similarity $\hat{\mathbf{E}}$. (For 100 repetitions of a simulated attack on DES with high noise levels, see Section 4 for a detailed description of such simulated attacks.) These graphs show the typical distribution of values for $\mathbf{S}$ and $\hat{\mathbf{E}}$ for correlated and/or uncorrelated time. They show that random $\hat{\mathbf{E}}$ values have a much higher chance of being close to the expected peak $\hat{e}_{c k, c t}=1$ and thus overtaking the correct value due to noise. 
We view $s_{i, j}$ and $\hat{e}_{i, j}$ as independent indicators for the 'likelyhood' of a key candidate, making their product a good choice for a combined indicator where only peaks which occur in both stand out. However, as $\mathbf{S}$ is less sensitive to noise we weight this indicator stronger than the indicator $\hat{\mathbf{E}}$ which we do by adding it again to the combined result resulting in the mixing function in Eq. (2). Note that the factors $\left(\frac{1}{2}, \frac{1}{2}\right)$ in this function are determined based on empirical study and may not be the optimal solution.

Remark 3. The EDPA attack as given in this section assumes that the correct peak has a positive sign. The attack can be easily adjusted to cope with unknown or negative signs (for the correct peak). E.g. when this sign is unknown, one can modify the mixing function in Step 3 so that $\left\{e_{i, j} \mid i=1 . . n, j=1 . . m\right\}$ that are of the same distance to their mean factorize their corresponding $s_{i, j}$ by the same amount.

\section{Evaluating ECPA Using Simulation}

In this section, we show results of simulated attacks on DES and AES using ECPA and CPA and compare the attacks using the security metrics described in Section 2 ,

\subsection{Simulation Setup}

We model the power consumption of a device as the summation of the Hammingweight of the processed intermediate value $v$, some constant value and noise (see e.g. [2]). The constant value is determined by the operation applied to $v$. The noise is a normally distributed variable with expectation 0 and some standard deviation $\sigma$.

For DES, we let $\sigma=10$ and simulate the power consumption for the first three rounds of an encryption. To be precise 28 power consumption values are generated per encryption round: 1 for the left half of the round input, 1 for the right half of the round input, 8 for the output of the expansion, 8 for the input of the S-box substitution, 8 for the output of the substitution, 1 for the output of the permutation, and 1 for the XOR of the permutation output and the left half of the round input.

For AES, we let ${ }^{2} \sigma=12$ and simulate the power consumption for the first round of an encryption. To be precise 108 power consumption values are generated: 16 each for the outputs of the initial KeyAddition, and the SubBytes, 12 for the output of the ShiftRows, 32 for the intermediate values of the MixColumns and 16 each for the outputs of the MixColumns, and the KeyAddition at the end of the first round.

\footnotetext{
${ }^{2}$ We choose $\sigma=10$ (resp. $\sigma=12$ ) for DES (resp. AES) to demonstrate the increase of success rate from 0 to 1 within query span $0 \leq q \leq 3000$, providing a good trade-off between the practicality of the simulation and the cost of the computation.
} 

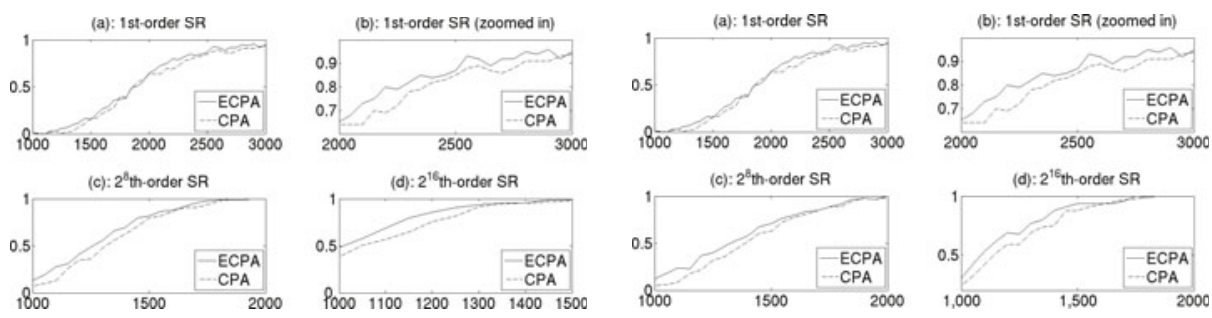

Fig. 4. DES with optimal sorting: SR for different number of queries

Fig. 5. DES with sub-optimal sorting: SR for different number of queries

To estimate SR and GE, we generate for each algorithm 100 sets of power traces, each containing 3000 simulated traces based on 3000 random plaintexts and one fixed randomly chosen key. Using statistical tests based on one random key is sufficient as, given the same amount of measurements, it is equally difficult to attack one key as an another key for DES and AES.

\subsection{Comparing ECPA and CPA on DES}

For attacks on DES, we target the eight 4-bit output of the S-boxes in the first round aiming to find the 48-bit round key used in this round. Hence, we perform eight sub-attacks each targeting a 6-bit sub-key used in an S-box. In this experiment, we refer to the SR (resp. the GE) of an attack as the probability (resp. the average cost) of recovering this 48-bit round key. In practice, the remaining 8 bits of the secret key can be found either by another SCA on the second encryption round or by a brute-force attack. Which method is used is immaterial for our comparison of ECPA and CPA.

We use both the optimal and the sub-optimal sorting algorithm for the evaluation of the success rate $s r_{\mathbf{g}_{q}}^{o}$ and the guessing entropies $g e_{\mathbf{g}_{q}}$ and $g e_{\mathbf{g}_{q}}^{o}$. Because the computational cost of sorting all the $2^{48}$ key candidates by the optimal sorting algorithm is too high to be practical, we limit the number of candidates to sort by this algorithm to $2^{16}$. Recall that checking a single candidate already requires a significant amount of work from an attacker (e.g. $2^{8}$ encryptions to find the remaining 8 bits).

Figures 4 and [5] show the SR of the ECPA attack and the CPA attack for various $q$ and $o$ using the two sorting algorithms. A $2^{8}$ th-order SR (resp. GE) corresponds to the success rate (resp. average cost) of a SCA attack provided that the adversary tests, on average, 2 candidates per S-box after the attack. A $2^{16}$ th-order SR (resp. GE) corresponds to the success rate (resp. average cost) of an attack provided that on average 4 candidates are tested per S-box.

For both sorting algorithms, ECPA results in a higher success rate than CPA in most of the cases. Comparing the graphs between the figures, we observe that for both sorting algorithms the 1st-order SRs are equal for every attack and every $q$. This is because both algorithms sort the same candidate the first. The 
higher-order SRs (see graphs (c) and (d) in Figures 4 and 5) differ per sorting algorithm. It is obvious that the optimal sorting algorithm almost always results in a higher success rate than the sub-optimal sorting algorithm. This gives us an empirical proof that the optimal sorting algorithm is indeed better than the sub-optimal sorting algorithm with respect to the success rate of an attack.

The guessing entropy with the ECPA and CPA attack are shown in Figures 6 and 7 for the two sorting algorithms used. Figure 6 show the GE of order $2^{16}$ while Figure 7 show the GE of order $2^{48}$, i.e. the full GE. The interesting range is roughly $2^{32} \geq \mathrm{GE} \geq 2^{8}$, as above this range a brute-force attack is too expensive and below this range the cost is too small for differences to matter. Both Figure 6 and Figure 7 show that ECPA improves on CPA, especially in this range.

\subsection{Comparing ECPA and CPA on AES}

For the attack on AES, we target the 8-bit output of the first S-box in the first round. Since the same S-box is used for every byte of the state and the power consumption caused by each of the $16 \mathrm{~S}$-box substitution is simulated based on the same function, we consider that it is as difficult to attack one S-box as attacking another in the same round. We, therefore, only look at the SR (resp. GE) of a sub-attack recovering one byte of the secret key of AES. An advantage in the sub-attack will translate to a proportional advantage in a full attack.

Figure 8 shows the 1st, the 2nd and the 4th-order SRs and the GE of the attacks. The 1st-order SR of the ECPA attack is approximately equal to the 1storder SR of the CPA attack. For the higher-order SRs, the advantage of ECPA over CPA also does not seem that large. However, when all are added in the GE, a clear benefit of ECPA can be seen. Note that this is only the advantage for the sub-attack and for a full attack, which repeats this attack 16 times, the advantage can grow quickly.

The advantage of ECPA over CPA is greater for DES S-boxes than for the AES S-box. Recall that the advantage of ECPA relies on the information leaked by the ghost peaks. The greater the ghost peaks caused structure in the analyzed instruction, the more information an ECPA attack gains in addition to a CPA attack. Because of their mathematical structure, with the same level of noise, a CPA attack on DES S-boxes leads to higher and more 'telling' ghost peaks,

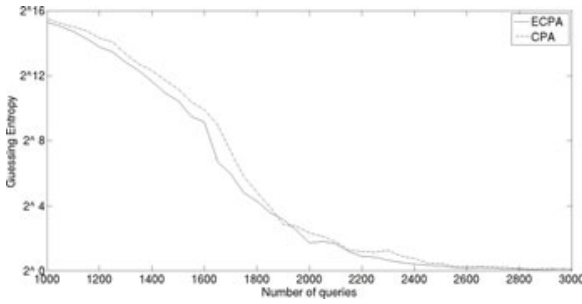

Fig. 6. DES with optimal sorting: $2^{16}$ thorder GE for different number of queries

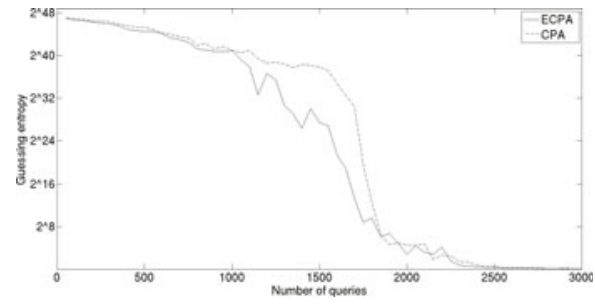

Fig. 7. DES with sub-optimal sorting: GE for different number of queries 

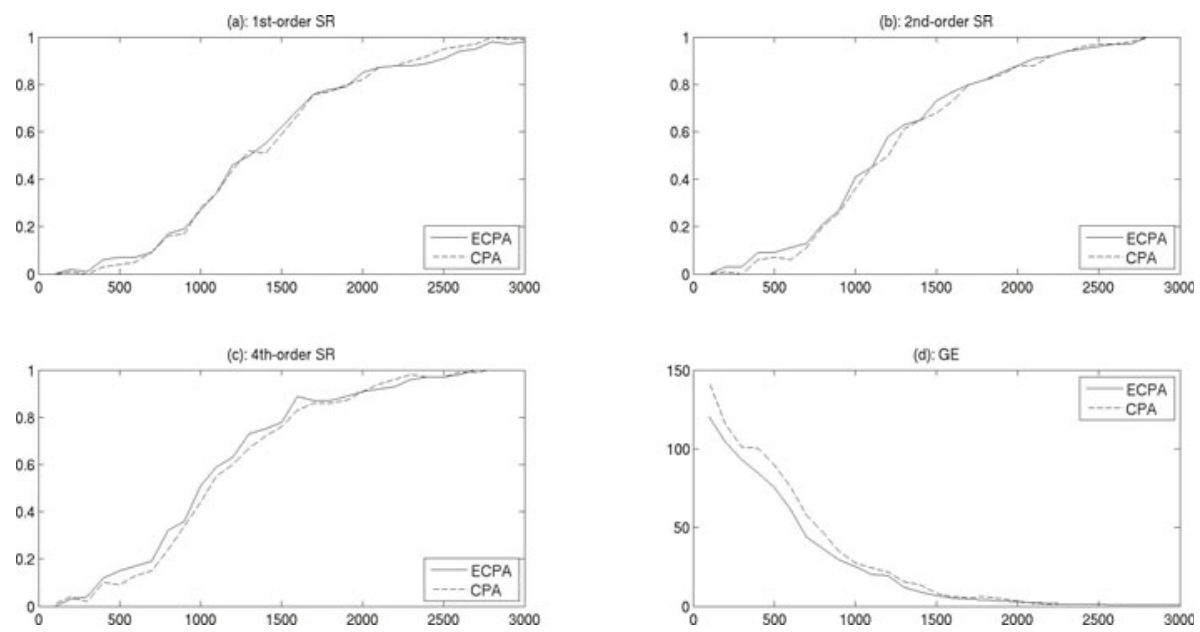

Fig. 8. AES: SR and GE for different number of queries

whereas the AES S-box would result in lower and more uniformly distributed peaks (see e.g. [14]). Hence, the ECPA attack is more effective on DES S-boxes.

\section{Evaluating ECPA Using Physical Experiments}

To validate our theoretical motivation, we performed CPA and ECPA attacks on two smart cards running a hardware DES and a software AES implementation respectively. The experiment shows that for both devices, with the tested number of traces, ECPA successfully finds the correct key while CPA does not 3 .

\subsection{Attacking a Hardware DES Implementation}

The attacked smart card has an 8-bit processor, with a DES accelerator containing two 32-bit registers. The hardware-implemented DES is countermeasure free. The DES accelerator is clocked at $30.76 \mathrm{MHz}$. For the power measurement, the oscilloscope collects samples at a frequency of $250 \mathrm{MHz}$ which are then compressed to one sample per internal clock period of the smart card, i.e. $30.76 \mathrm{MHz}$.

In the smart card that we attack, one round of DES encryption is completed within one clock cycle of the DES accelerator. Therefore, it is very difficult to exploit the leakage caused by the S-boxes substitution. Instead, we target the XOR of the 32-bit permutation output and the 32-bit left half of the round input that takes place at the end of the first round. Assuming that the result of this

${ }^{3}$ Though the comparison performed in this section considers both positive and negative peaks, advantage of ECPA over CPA can also be concluded even when only the positive peaks are regarded, which is the case when the power model of the attacked device is known beforehand. 

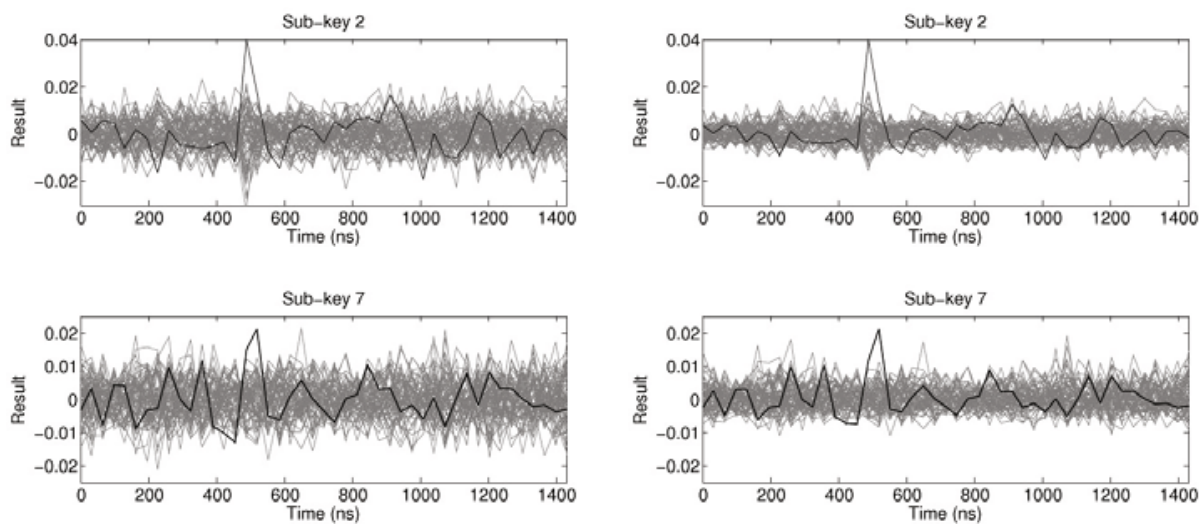

Fig. 9. CPA result of DES S-boxes 2 Fig. 10. ECPA result of DES S-boxes 2 and 7 and 7

$\mathrm{XOR}$ is written in the same register that previously contained the right half of the round input, we use the Hamming distance of these two 32-bit values as our power model. We use eight sub-attacks each targeting 6 key bits (used in the same S-box thus allowing prediction of 4 bits of the 32-bit intermediate value).

The attacks were performed using the same set of 23,000 power traces. We take sub-keys 2 and 7 as examples and show their attack results in Figures 9 and 10, where the results of the correct key candidate is plotted in black and the others are plotted in gray. The attack results for all sub-keys can be found in Appendix C. Though CPA and ECPA both reveal the correct sub-key 2, ECPA significantly lowers the incorrect peaks compared to CPA and therefore raises the confidence level of the correct key.

Sub-key 7 is ranked second by CPA but correctly found by ECPA, showing an improvement of ECPA on CPA. Decreasing the number power traces used, we find that for this sub-key the correct key is always sorted earlier by ECPA than by CPA (see Table 1). Experiments described in Table 11 show that on average ECPA approximately halfs the rank of the correct key compared to CPA. Concerning a global sorting that regards all the eight sub-keys, the difference of the ranking for sub-key 7 can add up to a much more significant difference of the overall ranking for the secret DES key.

Table 1. The rank of the correct sub-key 7 using decreasing numbers of power traces

\begin{tabular}{|c|c|c|c|c|c|c|c|c|c|}
\hline Number of Traces & $22 \mathrm{~K}$ & $21 \mathrm{~K}$ & $20 \mathrm{~K}$ & $19 \mathrm{~K}$ & $18 \mathrm{~K}$ & $17 \mathrm{~K}$ & $16 \mathrm{~K}$ & $15 \mathrm{~K}$ & $10 \mathrm{~K}$ \\
\hline CPA & 9 & 5 & 5 & 8 & 22 & 15 & 13 & 13 & 30 \\
ECPA & 3 & 2 & 3 & 5 & 18 & 14 & 6 & 3 & 17 \\
\hline
\end{tabular}


Table 2. The results of CPA (top) and ECPA (bottom) of an AES softwareimplementation. The attack results are written outside the brackets, and the sub-key candidates are written inside the brackets in hexadecimal numbers. The correct peaks and candidates are written in bold.

\begin{tabular}{|c|c|c|c|c|c|c|c|c|}
\hline ank & Byte 1 & Byte 2 & Byte & Byte 4 & Byte 5 & Byte 6 & Byte 7 & \\
\hline 1 & $.5845(8 d)$ & $4699(\mathrm{f5})$ & $.5186(91)$ & $.5227(54)$ & $.4659(6 \mathrm{e})$ & $.5832(\mathrm{c} 3)$ & $.4826(3 \mathrm{e})$ & $.5384(5 \mathrm{c})$ \\
\hline$\overline{2}$ & .435 & 8a) & $\overline{5)}$ & -.4468 & .461 & .472 & -.439 & -.438 \\
\hline$\overline{3}$ & & ). & & & & & $\overline{3 d)}$ & $-.4351(56)$ \\
\hline$\overline{4}$ & & & d) & -.430 & -.431 & & -.421 & \\
\hline tank & & $\mathrm{Byt}$ & & e 12 & & Byt & Byte 15 & Byte 16 \\
\hline 1 & .4788 & .4843 & .5219 & .4576 & .5445 & .5525 & $.4886(61)$ & $.5023(84)$ \\
\hline 2 & .4685 & -.465 & -.4220 & .448 & -.4399 & -.4194 & $-.4869(56)$ & $-.4395(\mathrm{fe})$ \\
\hline 3 & -.4289 & .4440 & -.4205 & -.4449 & .4160 & .4160 & $-.4532(72)$ & $-.4260(91)$ \\
\hline 4 & $.4211(\mathrm{cc})$ & $-.4087(41)$ & $.4199(\mathrm{a} 6)$ & $-.4190(5 b)$ & $-.4103(85)$ & \begin{tabular}{|l|}
$-.4103(85)$ \\
\end{tabular} & $.4527(\mathrm{e} 5)$ & $-.4228(\mathrm{a} 5)$ \\
\hline ank & Byte 1 & Byte 2 & Byte 3 & Byte 4 & Byte 5 & Byte 6 & Byte 7 & Byte 8 \\
\hline 1 & $.5845(8 d)$ & $.4612(\mathrm{f} 5)$ & $.5181(91)$ & $.5227(54)$ & $.4659(6 \mathrm{e})$ & $.5832(\mathrm{c} 3)$ & $.4887(3 \mathrm{e})$ & $.5234(5 c)$ \\
\hline 2 & $.3995(8 b)$ & $.3945(20)$ & $.4417(45)$ & $.4177(63)$ & $.4065(25)$ & $.4118(68)$ & $.4098(84)$ & $.3654(\mathrm{c} 8$ \\
\hline 3 & $.3912(\mathrm{~d} 8)$ & $.3914(7$ & $.3834(\mathrm{f}$ & .4108( & $.4043(2 \mathrm{e})$ & $.4002(\mathrm{f} 0)$ & $-.4005(\mathrm{~d} 8)$ & $.3643(5 \mathrm{~d})$ \\
\hline 4 & $\begin{array}{l}.3792(66) \\
\end{array}$ & $.3911(3 b)$ & $.3770(82)$ & $.3906(5 \mathrm{~d})$ & $.3980(\mathrm{~b} 3)$ & $.3802(76)$ & $.3935(45)$ & $.3643(85)$ \\
\hline ank & Byte 9 & Byte 10 & Byte 11 & Byte 12 & Byte 13 & Byte 14 & Byte 15 & Byte 16 \\
\hline 1 & $.4613(\mathrm{~d} 4)$ & $4659(6$ & $5219(7 \mathrm{c})$ & $.4418(\mathrm{c}$ & $.5445(\mathrm{ac})$ & $.5525(1 \mathrm{c})$ & $.4769(61)$ & $.4928(84)$ \\
\hline 2 & $.4224(\mathrm{f} 5)$ & .4186( & .3995( & .4296 & $.3871(\mathrm{c} 2)$ & $.3723(6 \mathrm{~b})$ & $.4271(\mathrm{e} 5)$ & $-.4147(9 \mathrm{~d}$ \\
\hline 3 & $.3950(\mathrm{df})$ & $.3600(1$ & $.3855(89)$ & $.3859(5 \mathrm{a})$ & $.3827(05)$ & $.3648(2 \mathrm{c})$ & $.4062(0 \mathrm{a})$ & 4037 (f5 \\
\hline 4 & $.3867(\mathrm{cc})$ & $.3597(\mathrm{fa})$ & $.3683(1 \mathrm{~b})$ & $\begin{array}{l}.3764(22) \\
\end{array}$ & $.3783(75)$ & $.3645(95)$ & $.3988(\mathrm{c} 0)$ & $3763(\mathrm{a} 8$ \\
\hline
\end{tabular}

\subsection{Attacking a Software AES Implementation}

The attacked smart card for the AES experiment shows quite a lot of side-channel leakage allowing us to suffice with 110 traces. Unlike the card we attacked in Section 5.1, this card does not have an accelerator embedded. The card works on its external clock, which is set at $4 \mathrm{MHz}$. The oscilloscope was set at frequency $200 \mathrm{MHz}$ and the signals were compressed to one sample per clock period of the card: $4 \mathrm{MHz}$.

For both ECPA and CPA attacks, we target the 16 bytes of the output of the S-boxes in the first encryption round. We use the Hamming weight of the bytes as our power model.

Table 2 lists the top candidates resulted from both attacks. The attacks use the same set of 110 traces. As in the previous experiment ECPA gives better results than CPA: all the correct sub-keys are ranked the first by ECPA and all the correct peaks stand out more significantly from the ghost peaks. The CPA attack fails to sort the correct candidate of byte 9 the first, resulting that the correct full key of AES will be sorted the 6th and the 9th candidate after the CPA attack according to the optimal and the sub-optimal sorting algorithms respectively. 


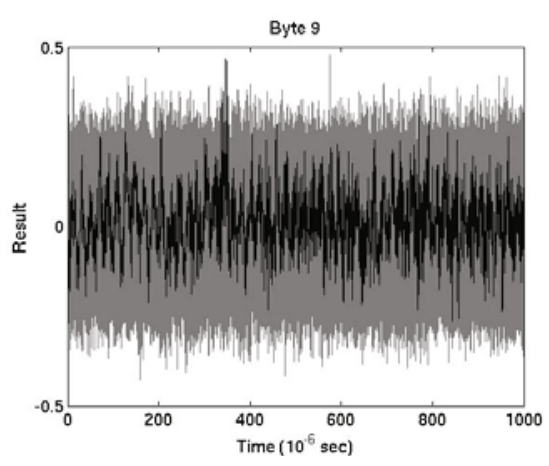

Fig. 11. CPA result of AES for byte 9 of AES

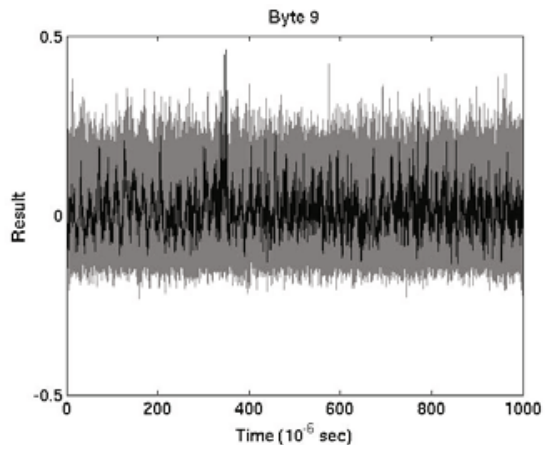

Fig. 12. ECPA result of AES for byte 9

Figures 11 and 12 plot the results of the attacks for byte 9, where the black (resp. gray) curves correspond to the correct (resp. incorrect) key candidates. Observe that the 'bandwidth' of the gray region is narrower in case of ECPA. Additionally, the highest ghost peak in CPA (Figure 11, $t \approx 570 \mu \mathrm{s}$ ), which is higher than the correct peak, gets reduced by ECPA (Figure $12, t \approx 570 \mu s$ ) and is now lower than the correct peak. Plots of the results for the other bytes can be found in Appendix C,

\section{Conclusion}

We introduce a new SCA attack - EDPA, which is able to extract more information from SCA leakage compared with a standard DPA attack and effectively diminishes ghost peaks using the information leaked by the ghost peaks themselves. We show with simulation and experiment that the extra information indeed improves the effectiveness of the attack. To help evaluate this effectiveness we introduce $o$-th order guessing entropy. We argue that the $o$-th order guessing entropy is more realistic and show that it is more practical with a scenario where the $o$-th order guessing entropy is possible but the original (full) guessing entropy cannot be used. Our results show a consistent advantage of EDPA over DPA but the advantage is not huge, which is to be expected as the attack simply improves the use of the same leaked information. Still, the limited computational overhead of an EDPA attack with respect to a DPA attack, and its efficiency in reducing the noise in the final result suggest that EDPA is at least an effective tool for further eliminating ghost peaks after an ambiguous DPA attack. Finally, since EDPA is based on the same leakage information as a DPA attack, countermeasures that prevent a standard DPA attack can also be used to thwart an ECPA attack. 


\section{References}

1. Bevan, R., Knudsen, E.: Ways to enhance differential power analysis. In: Lee, P.J., Lim, C.H. (eds.) ICISC 2002. LNCS, vol. 2587, pp. 327-342. Springer, Heidelberg (2003)

2. Brier, É., Clavier, C., Olivier, F.: Correlation power analysis with a leakage model. In: Joye, M., Quisquater, J.-J. (eds.) CHES 2004. LNCS, vol. 3156, pp. 16-29. Springer, Heidelberg (2004)

3. Clavier, C., Coron, J.-S., Dabbous, N.: Differential power analysis in the presence of hardware countermeasures. In: Paar, C., Koç, Ç.K. (eds.) CHES 2000. LNCS, vol. 1965, pp. 252-263. Springer, Heidelberg (2000)

4. Cormen, T., Leiserson, C., Rivest, R.: Introduction to Algorithms. The MIT Press, Cambridge (1990)

5. Coron, J.-S., Naccache, D., Kocher, P.C.: Statistics and secret leakage. ACM Trans. Embedded Comput. Syst. 3(3), 492-508 (2004)

6. Elmore, K.L., Richman, M.B.: Euclidean distance as a similarity metric for principle component analysis. American Meteorological Society 129(3), 540-549 (2001)

7. Gierlichs, B., Batina, L., Tuyls, P., Preneel, B.: Mutual information analysis. In: Oswald, E., Rohatgi, P. (eds.) CHES 2008. LNCS, vol. 5154, pp. 426-442. Springer, Heidelberg (2008)

8. Kocher, P.C.: Timing attacks on implementations of diffie-hellman, rsa, dss, and other systems. In: Koblitz, N. (ed.) CRYPTO 1996. LNCS, vol. 1109, pp. 104-113. Springer, Heidelberg (1996)

9. Kocher, P.C., Jaffe, J., Jun, B.: Differential power analysis. In: Wiener, M. (ed.) CRYPTO 1999. LNCS, vol. 1666, pp. 388-397. Springer, Heidelberg (1999)

10. Mangard, S.: Hardware countermeasures against dpa - a statistical analysis of their effectiveness. In: Okamoto, T. (ed.) CT-RSA 2004. LNCS, vol. 2964, pp. 222-235. Springer, Heidelberg (2004)

11. Mangard, S., Oswald, E., Popp, T.: Power Analysis Attacks: Revealing the Secrets of Smart Cards. In: Advances in Information Security. Springer, Heidelberg (2007)

12. Messerges, T.S., Dabbish, E.A., Sloan, R.H.: Examining smart-card security under the threat of power analysis attacks. IEEE Trans. Computers 51(5), 541-552 (2002)

13. Orfanidis, S.J.: Introduction to signal processing. Prentice-Hall, Inc., Upper Saddle River (1995)

14. Pan, J., den Hartog, J.I., de Vink, E.P.: An operation-based metric on cpa resistance. In: Jajodia, S., Samarati, P., Cimato, S. (eds.) SEC, International Federation for Information Processing, pp. 429-443. Springer, Boston (2008)

15. Quisquater, J.-J., Samyde, D.: Electromagnetic analysis (ema): Measures and counter-measures for smart cards. In: Attali, I., Jensen, T.P. (eds.) E-smart 2001. LNCS, vol. 2140, pp. 200-210. Springer, Heidelberg (2001)

16. Standaert, F.-X., Gierlichs, B., Verbauwhede, I.: Partition vs. comparison sidechannel distinguishers: An empirical evaluation of statistical tests for univariate side-channel attacks against two unprotected cmos devices. In: Lee, P.J., Cheon, J.H. (eds.) ICISC 2008. LNCS, vol. 5461, pp. 253-267. Springer, Heidelberg (2009)

17. Standaert, F.-X., Malkin, T., Yung, M.: A unified framework for the analysis of side-channel key recovery attacks. In: Joux, A. (ed.) EUROCRYPT 2009. LNCS, vol. 5479, pp. 443-461. Springer, Heidelberg (2009)

18. van Woudenberg, J.G.J., Witteman, M.F., Bakker, B.: Improving differential power analysis by elastic alignment (2009), http://www.riscure.com/fileadmin/images/Docs/elastic_paper.pdf 


\section{A The Sorting Algorithms}

In this section we present the two key sorting algorithms. We take a fixed number of sub-key candidates for each sub-attack, and assume all sub-attacks use the same set of traces and that none of the peaks are exactly the same. All of these assumptions can be easily removed at the cost of complicating the notation.

\section{A.1 Pre Processing Sub-SCA Results}

Depending on the implementation, the measurement, and other factors, the amount of exploitable information can be different for different sub-attacks. Rather than using the actual SCA result, a normalized SCA result gives better estimation of relative likelihood, which is what we want to sort on. E.g. Consider two sub-attacks where the top two candidates of the attacks have peaks $(0.8,0.7)$ and $(0.2,0.1)$, respectively. Clearly we want to try the second candidate from attack $1(0.7)$ before that of attack $2(0.1)$ as it seems more likely to be the correct key.

Hence, the sorting algorithms presented in this section take as reference the relative SCA value of a key candidate that is with respect to the highest SCA value within the same sub-attack. That is, we sort a key candidate by the ratio between the SCA result of a key candidate and the highest SCA value resulted from the same attack. See Table 3 for an description. We do not give an implementation of this computation since it is relatively trivial.

Table 3. Pre processing sub-SCA results

\begin{tabular}{l}
\hline \hline INPUT: A list of SCA results $r_{i, j}^{(a)}(a=1 . . A, i=1 . . n, j=1 . . m)$, where $A$ is the number \\
of sub-attacks performed, $n$ is the number of sub-key candidates per sub-attack and $m$ \\
is the number of leakage samples per power trace. \\
OUTPUT: Normalized maximal SCA peaks for each sub-key candidate $r r_{i}^{(a)}=$ \\
$p k_{i}^{(a)} / \max \left\{p k_{i^{\prime}}^{(a)} \mid i^{\prime}=1 . . n\right\}$ where $p k_{i}^{(a)}=\max \left\{\left|r_{i, j}^{(a)}\right| \mid j=1 . . m\right\}$.
\end{tabular}

\section{A.2 The Sub-optimal Sorting Algorithm}

According to the normalized SCA peaks, the sub-optimal algorithm first rates every sub-key candidates with its global ranking concerning all sub-attacks. The vector $\mathbf{g}$ is initialized with the top candidate from every sub-attack: $\left(k_{\max }^{(1)}, \ldots\right.$, $\left.k_{\max }^{(A)}\right)$. Next, the rest of sub-key candidates are brought into consideration in the order of their global ratings. Before considering any combination which uses a sub-key with a global rating $x$, the algorithm will tests all candidates whose sub-keys all have a global ranking lower than $x$. To add a sub-key $k_{i}^{(a)}$ into $\mathbf{g}$, we first find all the items in $\mathbf{g}$ that use $k_{\max }^{(a)}$, then we replace $k_{\max }^{(a)}$ with $k_{i}^{(a)}$ and append the updated items into $\mathbf{g}$ in the order of their original items in $\mathbf{g}$. 
Table 4. The sub-optimal sorting algorithm

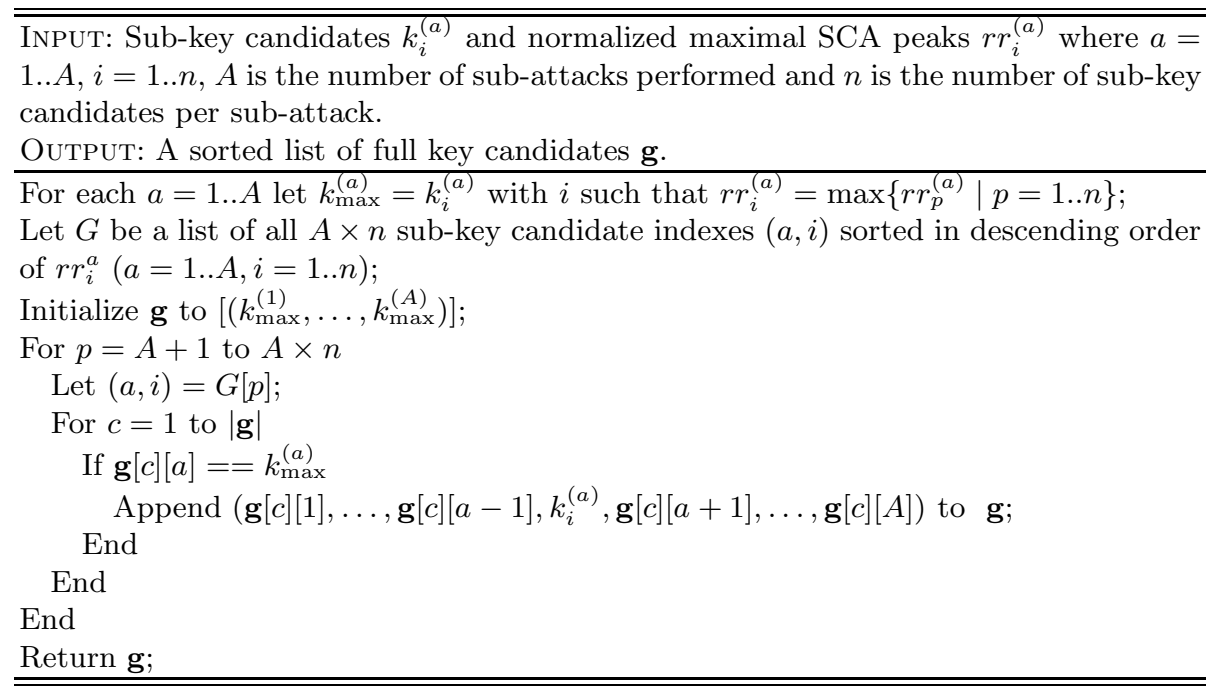

To estimate the SR and GE of an attack with a known key, we do not need the whole vector $\mathbf{g}$ but only the position of the real key in this vector. This ranking $c r$ of the correct key can be computed by Eq (3).

$$
c r=1+\sum_{a=1}^{A} \prod_{\substack{b=1, b \leq_{G} a}}^{A} \#\left\{i \mid i=1 . . n,(b, i)<_{G}\left(a, c k_{a}\right)\right\} .
$$

There we write $c k_{a}$ for the index of correct key candidate in sub-attack $a(a=$ $1 . . A)$, i.e. the correct key is $\left(k_{c k_{1}}^{(1)}, \ldots, k_{c k_{A}}^{(A)}\right)$, we write $(a, i)<_{G}\left(a^{\prime}, i^{\prime}\right)$ if $(a, i)$ appears before $\left(a^{\prime}, i^{\prime}\right)$ on list $G$ and we write $a<_{G} b$ if $\left(a, c k_{a}\right)<_{G}\left(b, c k_{b}\right)$ i.e. if the correct index of sub-attack $a$ appears before the correct index of sub-attack $b$ on list $G$.

\section{A.3 The Optimal Sorting Algorithm}

The optimal sorting algorithm (in Table 5) outputs the full key candidates based on their monotonically non-increasing sum (for $a=1 . . A$ ) of normalized sub-SCA results $\operatorname{rr}_{i}^{(a)}$.

The algorithm works by creating a sub-key-specific set of candidates $L$ sorted by decreasing SCA value. Next, a key candidate indexes set $H$ is initialized with the best candidate, which by definition consists of the top sub-key in each $L^{(a)}$.

The set $H$ is now iteratively updated by first removing the candidate $h$ with the highest sum and adding the corresponding key to g. The candidate $h$ is now expanded to all its successors, represented by $E(h)$. This set contains for each sub-key the next best candidate. The set $E(h)$ contains $A$ elements unless all $n$ 
Table 5. The optimal sorting algorithm

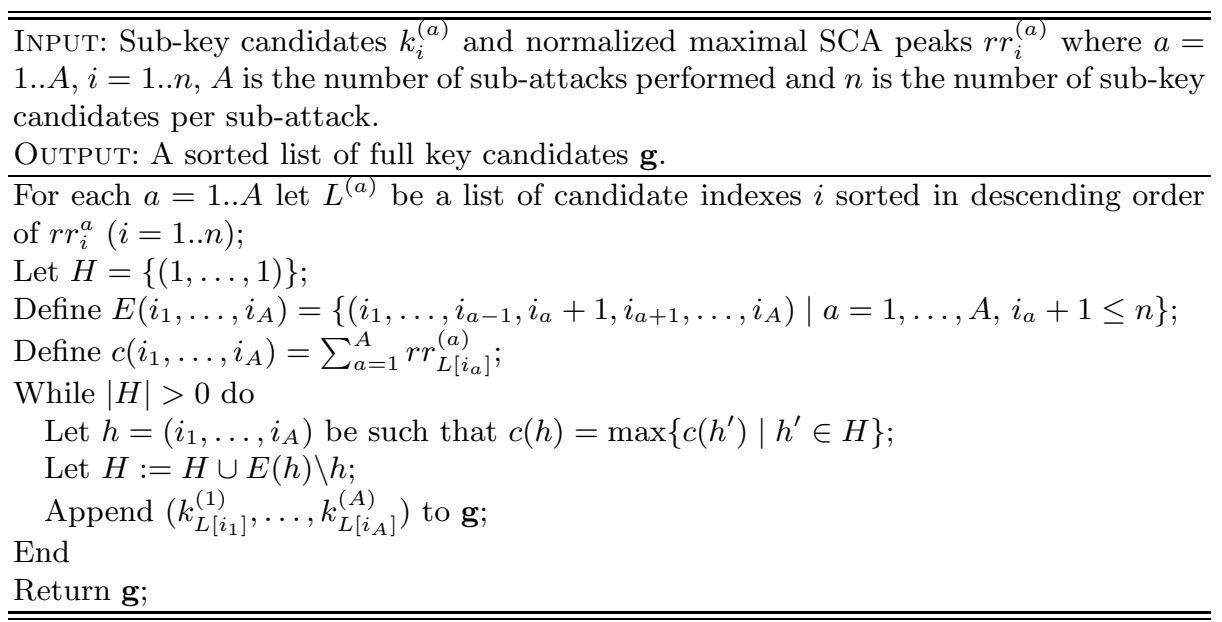

candidates for a sub-key have already been tried. Next, $E(h)$ is added to the set $H$.

Note that $H$ is a set, and can therefore not contain duplicate items. Because of the construction of the search, the entire space is guaranteed to be visited; the $\mathbf{g}$ contains all possible keys without duplicates. By sorting vectors $L^{(a)}$ and incrementally walking through the elements, we also guarantee a non-increasing sum.

The complexity of this sorting algorithm is $O(N \log N)$, with $N=n^{A}$ being the number of possible keys. The loop of the algorithm walks though all $N$ candidates. As $|H|<N$, the set query max, insertion and deletion are $O(\log N)$ if implemented using e.g. a red-black tree 4 .

We are not aware of an effective method (i.e. polynomial in the key size $n$ ) to determine the ranking of the correct key for this algorithm.

\section{B Block Diagram of ECPA}

The block diagram of the ECPA attack is plotted in Figure 15

\section{Graphical Presentation of the Practical Experiment Results}

In this section we show the plotting of the attack results from the physical experiments described in Section 5], where a DES hardware implementation and an AES software implementation were attacked by both ECPA and CPA.

In the experiment on the DES implementation, the same set of 23,000 power traces were used in both attacks to reveal the sub-keys used in the first encryption 

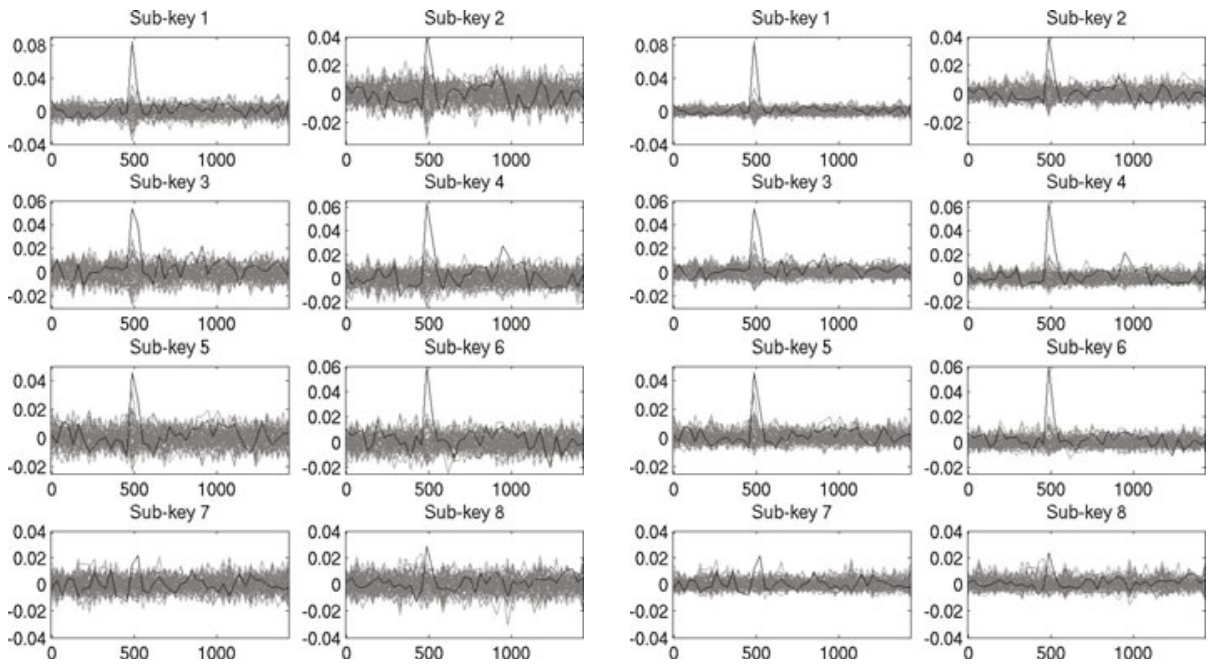

Fig. 13. CPA result of a DES implementation: $\mathrm{x}$-axis is time (ns), $\mathrm{y}$-axis is correlation

Fig. 14. ECPA result of a DES implementation: $\mathrm{x}$-axis is time (ns), $\mathrm{y}$-axis is correlation

round. Figures 13 and 14 show the results of the attacks. Every graph in these figures shows the results of 64 sub-key candidates that were involved in one of the S-box substitutions. In case of the AES experiment, 110 power traces were used to reveal the first round key of an AES encryption. Figures 16 and 17 show the results of the attacks for all key candidates and a selected range of samples in time, where each graph corresponds to one key byte.

In all the graphs shown in this section, the black curves correspond to the correct key candidates and the gray curves correspond to the incorrect key candidates. One can observe that for every attacked sub-key the gray band formed by the ghost peaks is reduced by ECPA compared to CPA. 


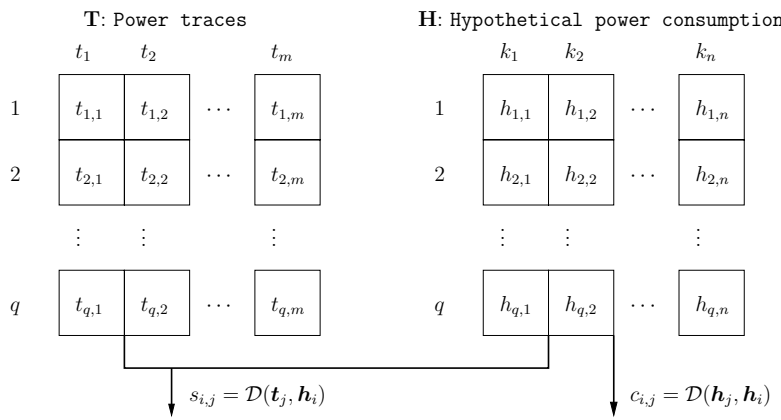

S: DPA result

C: Inter-data correlation

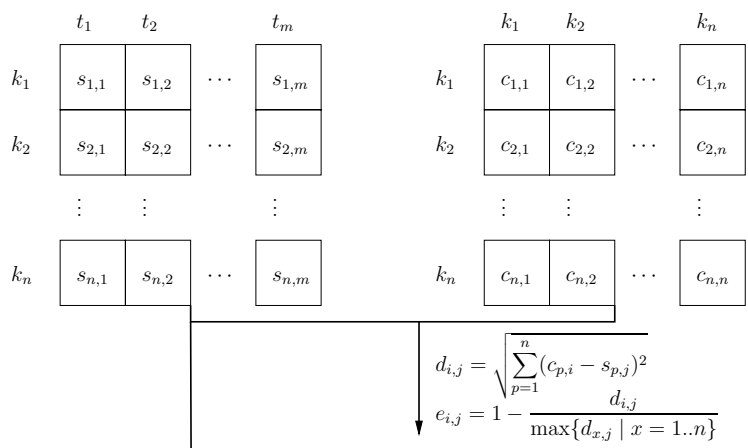

E: Euclidean similarity

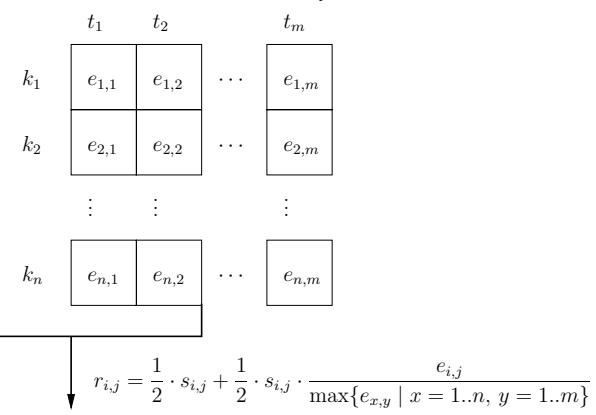

R: EDPA result

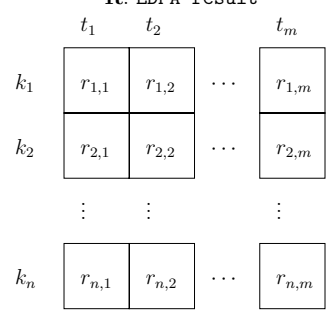

Fig. 15. Block diagram illustrating the last steps of an ECPA attack 

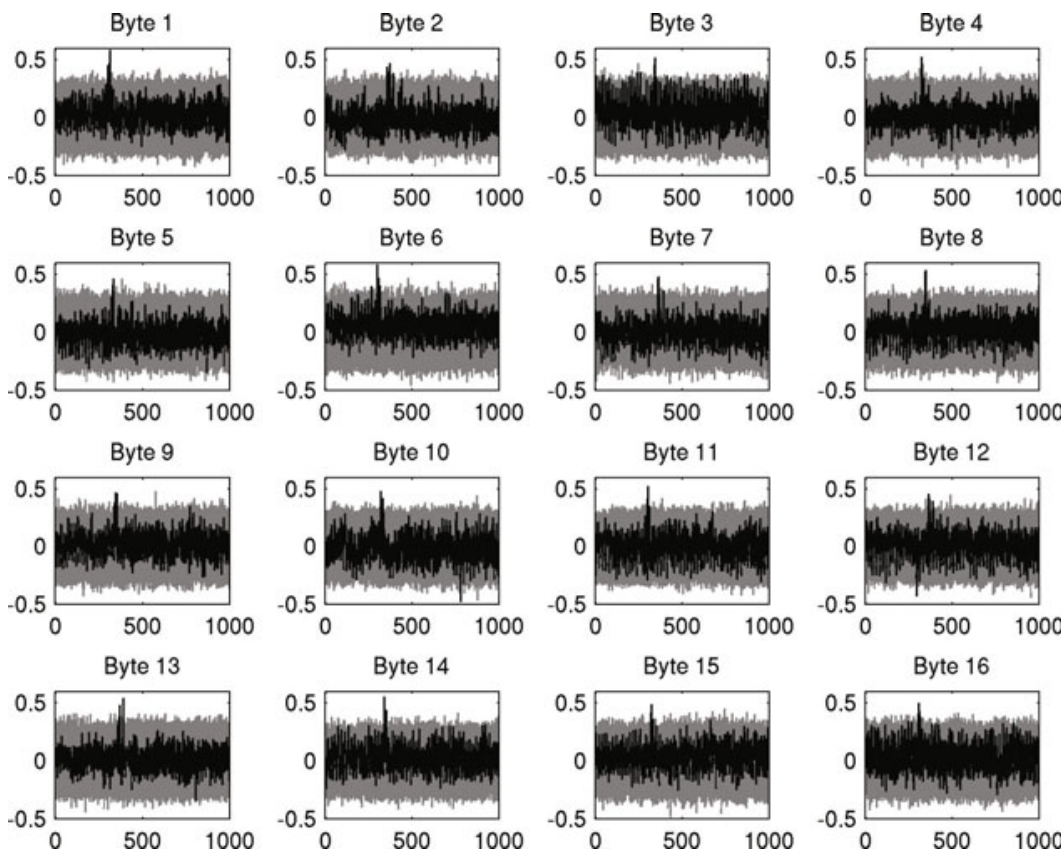

Fig. 16. CPA result on AES: $\mathrm{x}$-axis is time $(\mu \mathrm{s})$, y-axis is correlation
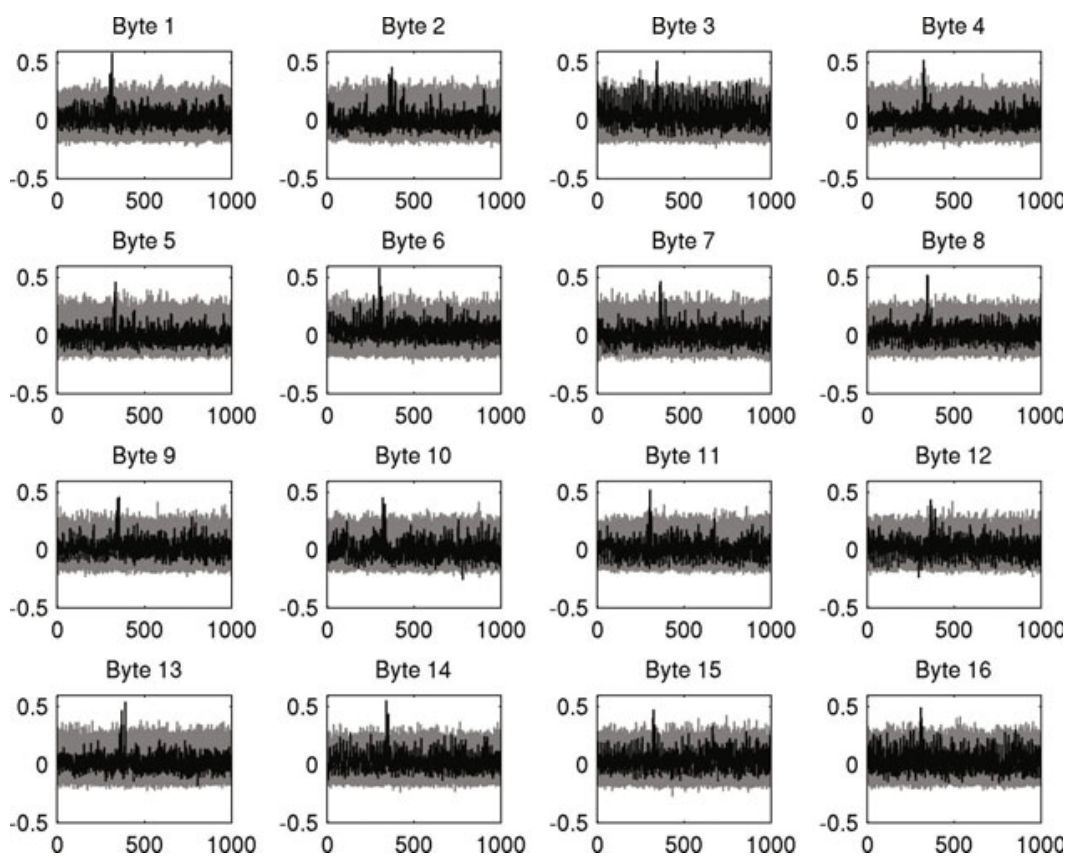

Fig. 17. ECPA result on AES: $\mathrm{x}$-axis is time $(\mu \mathrm{s}), \mathrm{y}$-axis is correlation 